\title{
Charge-based precipitation of extracellular vesicles
}

\author{
MARIA CHIARA DEREGIBUS ${ }^{1}$, FEDERICO FIGLIOLINI ${ }^{2}$, SERGIO D'ANTICO $^{3}$, PAOLA MARIA MANZINI $^{3}$, \\ CHIARA PASQUINO ${ }^{2}$, MICHELA DE LENA ${ }^{2}$, CIRO TETTA $^{4}$, MARIA FELICE BRIZZI $^{1}$ and GIOVANNI CAMUSSI ${ }^{1}$ \\ ${ }^{1}$ Department of Medical Sciences, University of Turin, ${ }^{2} 2 \mathrm{i} 3 \mathrm{~T}$ - Scarl. - Molecular Biotechnology Center (MBC), \\ University of Turin; ${ }^{3}$ Blood Bank, A.O.U. Città della Salute e della Scienza, \\ I-10126 Turin, Italy; ${ }^{4}$ Unicyte AG, 6370 Oberdorf, Switzerland
}

Received May 13, 2016; Accepted August 3, 2016

DOI: $10.3892 / \mathrm{ijmm} .2016 .2759$

\begin{abstract}
Vesicular-mediated communication between cells appears critical in many biological processes. Extracellular vesicles (EVs) released from healthy and diseased cells are involved in a network of exchange of biologically active molecules. Since EVs present in biological fluids carry the signature of the cell of origin, they are potential biomarkers for ongoing physiological or pathological processes. Despite the knowledge on EV biology accrued in recent years, techniques of EV purification remain a challenge and all the described methods have some advantages and disadvantages. In the present study, we described a method based on charge precipitation of EVs from biological fluids and from cell supernatants in comparison with the differential ultracentrifugation, which is considered the gold standard for EV purification. The analysis of $\zeta$-potential revealed that EVs have a negative charge that allows the interaction with a positively charged molecule, such as protamine. Protamine was shown to induce EV precipitation from serum and saliva and from cell culture media without the need for ultracentrifugation. EV resuspension was facilitated when protamine $(\mathrm{P})$ precipitation was performed in the presence of PEG 35,000 Da (P/PEG precipitation). The recovery of precipitated EVs evaluated by NanoSight analysis was more efficient than that obtained by ultracentrifugation. By electron microscopy the size of EVs was similar after both methods were used, and the expression of CD63, CD9 and CD81 exosomal markers in the P/PEG-precipitated EVs indicated an enrichment in exosomes. The RNA recovery of P/PEG-precipitated EVs was similar to that of EVs isolated by ultracentrifugation. In addition, P/PEG-precipitated EVs retained the biological activity in vitro as observed by the induction of wound closure by keratinocytes and of prolif-
\end{abstract}

Correspondence to: Professor Giovanni Camussi, Department of Medical Sciences, University of Turin, Corso Dogliotti 14, I-10126 Torino, Italy

E-mail: giovanni.camussi@unito.it

Abbreviations: EVs, extracellular vesicles; HLSCs, human liver stem cells

Key words: exosomes, microvesicles, miRNAs, mRNA eration of tubular epithelial cells. In conclusion, charge-based precipitation of EVs has the merit of simplicity and avoids the requirement of expensive equipments and may be used for the efficient isolation of EVs from small biological samples.

\section{Introduction}

Small vesicles released from cells have recently emerged as important mediators of inter-cellular communication. These vesicles that have been termed extracellular vesicles (EVs) are inclusive of exosomes released from the endosomal cell-membrane compartment and of microvesicles released from the cell surface by plasma membrane budding. The EV content of proteins, lipids and nucleic acids varies with the cell of origin and after incorporation into recipient cells, they may transfer information that may change the phenotype and function of recipient cells (1-3). Previous studies have addressed the role of EVs in physiological and pathological conditions based on their biological activity and molecular constituents (1-3). Additionally, since EVs retain the signature of the cell of origin and are present in all body fluids, their potential use as diagnostics in different pathological conditions has been suggested. A fundamental issue remains on how to isolate EVs from cultured cells in order to study their biological functions or from biological fluids for diagnostic purposes. Since fetal bovine serum frequently used for cell culture is enriched in EVs, in vitro experiments require the use of serum depletion of EVs (4). By contrast, the isolation of EVs from body fluids leads to the management of the complexity due to the concomitant presence of EVs of different cell origin. Therefore, in order to identify a potential biomarker it is critical to discriminate cell origin on the base of EV molecular expression or content by proteomic or genomic analysis.

Following removal of cell debris by centrifugation, the three main methods used for isolation of EVs include differential ultracentrifugation in the absence or presence of sucrose gradient, size exclusion chromatography, and immune affinity. These methods have some advantages mainly associated with the possibility to discriminate between different EV populations and concerns related to the risk to damage vesicles during purification with loss of biological activity, the need of a sufficiently large sample and the efficiency of isolation (reviewed in ref. 5). In addition, polymeric precipitation has been suggested as an alternative method mainly focused on the evaluation of RNA and protein content (6). The methods of polymeric 
precipitation are based on the formation of a mesh-like net that embeds EVs with a size ranging from 60 to $180 \mathrm{~nm}$. These methods may be applied to culture media or to body fluids. In particular, polymeric precipitation methods may have the advantage in the detection of biomarkers in vesicles derived from small biological samples.

The aim of the present study was to investigate the possibility to implement polymeric precipitation with a charge-based precipitation of EVs. For this purpose, we first measured the charge of EVs from different biological sources. Taking into account the EV-negative charge, we precipitated EVs in the presence of positively charged protamine in a polymeric matrix and compared the efficiency with ultracentrifugation in terms of yield of recovered vesicles, efficiency of RNA extraction, exosomal protein expression and biological activity.

\section{Materials and methods}

Biological samples. Saliva was obtained from adult normal volunteers $(n=5)$. The study of exosomes/microvesicles in saliva and serum of healthy human volunteers was approved by the Internal Ethics Committee of the Molecular Biotechnology Center. Human serum from healthy blood donors $(n=5)$ was provided by the Blood Bank of Città della Salute e della Scienza di Torino, after informed consent and approval by the internal Review Board of Blood Bank were obtained.

Adult human liver stem cells (HLSCs). HLSCs were isolated from human cryopreserved normal adult hepatocytes (Lonza, Basel, Switzerland), cultured and characterized as previously described (7). Briefly, hepatocytes were first cultivated for 2 weeks in Hepatozyme-SFM medium (Gibco, Grand Island, NY, USA), then in $\alpha$-MEM/EBM-1 (3:1) (Invitrogen, Carlsbad, CA, USA) media added with HEPES (12 mM, pH 7.4), L-glutamine $(5 \mathrm{mM})$ penicillin $(50 \mathrm{IU} / \mathrm{ml})$, streptomycin $(50 \mu \mathrm{g} / \mathrm{ml})$ (all from Sigma, St. Louis, MO, USA), and fetal calf serum (FCS) (10\%) (Invitrogen). The cells were expanded and characterized. The characterization of HLSCs by cytofluorimetric analysis demonstrated the expression of the mesenchymal stem cell markers but not of the endothelial and hematopoietic markers as previously described (7). HLSCs also expressed $\alpha$-fetoprotein, human albumin, vimentin and nestin resident stem cell markers, but not CD34, CD117 and cytokeratin 19 oval cell markers (7). In addition, HLSCs were positive for the Nanog, Sox 2, Oct4 and SSEA4 embryonic stemcell markers (8).HLSCsunder appropriate culture conditions underwent endothelial, osteogenic and hepatic differentiation (7).

Keratinocytes. Keratinocytes (HaCaT) were purchased and cultured with KBM-gold basal medium (Lonza, Basel, Switzerland) at $37^{\circ} \mathrm{C}$ with $5 \% \mathrm{CO}_{2}$. The cells were seeded at density $3.5 \times 10^{2}$ cell $/ \mathrm{cm}^{2}$, using $1 \mathrm{ml}$ of medium $/ \mathrm{cm}^{2}$ and subcultured when cell confluence was $70-80 \%$. Briefly, flasks were washed with HEPES buffer saline solution, incubated with trypsin solution for $6 \mathrm{~min}$ and then trypsin was neutralized with medium containing $10 \%$ FCS. If the cells were not completely detached within $7 \mathrm{~min}$, incubation with trypsin was repeated.

Renal tubular epithelial cells (TEC). TEC line immortalized by infection with a hybrid Adeno5/SV40 virus was previously devel- oped by Cantaluppi et al (9). Cells were grown using Dulbecco's modified Eagle's medium(DMEM) (Lonza) containing 10\% FCS (Gibco) and $2 \mathrm{mM}$ glutamine (Life Technologies, Carlsbad, CA, USA). TEC showed negative staining for von Willebrand factor, minimal staining for desmin and vimentin, and marked staining with antibodies directed to cytokeratins and actin. TEC was also positive for markers of fully differentiated proximal TEC such as alkaline phosphatase, aminopeptidase A and megalin.

Isolation of EVs. EVs were purified from the HLSC culture media, human serum and saliva. EVs isolated from the supernatants of HLSCs $\left(2 \times 10^{6}\right.$ cells/T75) were obtained after $24 \mathrm{~h}$ culture in RPMI-1640 deprived of FCS. At the time of EV isolation, 97-99\% of cells was viable by trypan blue exclusion assay, although the TUNEL assay did not detect apoptotic cells.

Saliva samples $(5 \mathrm{ml})$ were collected in sterile tubes and kept in ice during harvest. Serum samples were collected from healthy donors using serum separating tubes (BD) and centrifuged at $1,500 \mathrm{x} \mathrm{g}$ for $15 \mathrm{~min}$.

Prior to the isolation procedures, HLSC supernatant, saliva and serum samples were submitted to two centrifugations at 3,000 x g for $20 \mathrm{~min}$ to remove cell debris and other contaminants. The saliva samples were diluted 1:1 with phosphate-buffered saline (PBS) and filtered with $0.22 \mu \mathrm{m}$ filters.

Differential ultracentrifugation. Following the removal of cell debris and apoptotic bodies by two centrifugations at 3,000 x g for $20 \mathrm{~min}$, EVs were purified as previously described by Théry et al (10) by a first ultracentrifugation at $10,000 \times \mathrm{g}$ followed by ultracentrifugation at $100,000 \mathrm{xg}$ for $1 \mathrm{~h}$ at $4^{\circ} \mathrm{C}$ (Beckman Coulter Optima L-90K; Beckman Coulter, Fullerton, CA, USA).

Charge-based precipitation. In preliminary experiments, samples were incubated with various doses of protamine (1.0, $0.5,0.25$ and $0.1 \mathrm{mg} / \mathrm{ml}$ ) to determine the optimal protamine concentration. The biological samples ready for the precipitation procedure were transferred in sterile vials and added with the protamine (P) (Sigma)/Polyethylene glycol (PEG 35,000; Merck KGaA, Darmstadt, Germany) precipitation solution (P/PEG) (1 volume precipitation solution:4 volume sample). Control P or PEG 35,000 alone (PEG) served as the controls. The composition of precipitation solution was $0.2 \mathrm{~g}$ PEG 35,000 (Merck KGaA) and $1 \mathrm{mg}$ protamine chloride/ml (Sigma) of distilled water.

After overnight incubation at $4^{\circ} \mathrm{C}$, the mixture was centrifuged at $1,500 \mathrm{x}$ g for $30 \mathrm{~min}$ at $22^{\circ} \mathrm{C}$ and the supernatant was discarded. The pellet was re-suspended in the appropriate buffer to study biological activities or in lysis buffer for RNA extraction and western blot analysis.

Toremove the lipoproteins, Sephadex G-100 (GEHealthcare Bio-Sciences AB, Uppsala, Sweden) spin columns were prepared and samples were centrifuged at 1,000 $\mathrm{x} g$ for $1 \mathrm{~min}$. EVs were recovered in the void volumes.

Measure of EV charge. The analysis was performed by Zeta-sizer nanoinstrument (size range, $0.3 \mathrm{~nm}-10 \mu \mathrm{m}$; Malvern Instruments SA, Vénissieux, France). The $\zeta$ potential (slipping plane) was generated at $\mathrm{x}$ distance from the particle indicating the degree of electrostatic repulsion between adjacent, simi- 
larly charged particles in a dispersion. Negative $\zeta$-potential indicated a high grade of dispersion across the particles.

Nanoparticle tracking analysis (NTA). NanoSight LM10 (Malvern Instruments, Malvern, UK) was used to analyze the concentration and size distribution of EVs by means of the NTA software (Malvern Instruments SA). The Brownian movements of EVs present in the sample subjected to a laser light source were recorded by a camera and converted into size and concentration parameters by NTA through the Stokes-Einstein equation [https://en.wikipedia.org/wiki/ Einstein_relation_(kinetic_theory)].

Transmission electron microscopy. Transmission electron microscopy was performed on EVs isolated by ultracentrifugation or charge-based precipitation resuspended in PBS, placed on 200 mesh nickel formvar carbon-coated grids (Electron Microscopy Science, Hatfield, PA, USA) and left to adhere for $20 \mathrm{~min}$. The grids were then incubated with $2.5 \%$ glutaraldehyde containing $2 \%$ sucrose and after washings in distilled water the EVs were negatively stained with NanoVan (Nanoprobes, Yaphank, NK, USA) and observed using a Jeol JEM 1010 electron microscope (Jeol, Tokyo, Japan).

Western blot analysis. Protein content of the EV preparations was quantified using the Bradford method (Bio-Rad, Hercules, CA, USA). Protein samples were separated by $4-15 \%$ gradient sodium dodecyl sulfate-polyacrylamide gel electrophoresis and subjected to immunoblotting with rabbit polyclonal antibodies (1:1000 dilutions) anti-CD9 (Cat. no. ab155825), CD63 (Cat. no. ab199921), CD81 (Cat. no. ab109201), anti-apolipoprotein B100 (ApoB100; 1:5,000 dilution; Cat. no. ab20737) and goat polyclonal antibody anti-apolipoprotein A1 (ApoA1; 1:5,000 dilution; Cat. no. ab7613) (Abcam, Cambridge, UK). The protein bands were visualized using an enhanced chemiluminescence (ECL) detection kit and ChemiDoc ${ }^{\mathrm{TM}}$ XRS + System (Bio-Rad). Cell and EV lysates were loaded at concentrations of $30 \mu \mathrm{g} / \mathrm{well}$.

RNA extraction. The mirVana RNA isolation kit (Thermo Fisher Scientific, Waltham, MA, USA) was used to extract total RNA from EVs following the manufacturer's protocol. RNA was spectrophotometrically quantified (NanoDrop ND-1000; NanoDrop, Wilmington, DE, USA).

miRNA and $m R N A$ profiling by quantitative PCR. Quantitative PCR was carried out as previously described (11) using a 48-well StepOne ${ }^{\mathrm{TM}}$ Real-Time system (Applied Biosystems, Waltham, MA, USA). In brief, $0.2 \mathrm{mg}$ RNA was first reverse-transcribed using a miScript reverse transcription kit (Qiagen, Valencia, CA, USA). Subsequently, 3 ng of cDNA in triplicate were employed to identify and measure significant miRNAs performing RT-qPCR using a miScript SYBR-Green PCR kit (Qiagen, Valencia, CA, USA). miRNA-specific primers to hsa-miR-16 (5'-TAG CAG CAC GTA AAT ATT GGC G-3'), 29a (5'-TAG CAC CAT CTG AAA TCG GTT A-3'), 99b (5'-CCC GTA GAA CCG ACC TTG C-3'), 191 (5'-CAA CGG AAT CCC AAA AGC AG-3'), 223 (5'-TGT CAG TTT GTC AAA TAC CCC A-3') were used in separate reactions. The RNU44 (purchased by Qiagen) and RNU48 (5'-AAC TCT GAG TGT GTC GCT GAT G-3') snoRNAs
Table I. Analysis of $\zeta$ potential on biological samples.

\begin{tabular}{lc}
\hline potential of EVs & $\mathrm{mV}$ \\
\hline HLSC & -13.800 \\
Serum & -7.825 \\
Saliva & -8.54
\end{tabular}

EVs, extracellular vesicles; HLSCs, human liver stem cells.

served as the positive controls and $10 \mu 1$ of water were used as the negative control in place of the RNA.

RT-qPCR analysis was also performed for the presence of mRNA of ID1 (F, 5'-GGC GGC ATG CGT TCC-3' and R, 5'-TTG TTC TCC CTC AGA TCC GG-3') in serum, Annexin A1 (F, 5'-CGG AAC GCT TTG CTT TCT CTT and R, 5'-CAA GGC CCT GGC ATC TGA-3') in saliva and DCR1 (F, 5'-CGT TAT CAT TCC AAG ATA TCG CAA-3' and R, 5'-GGG TAA GAT CAG TGT ACA CAT CAG CT-3') in HLSC EVs.

Cell proliferation assays. Immortalized TEC was seeded at a density of $3 \times 10^{3}$ cells/well in 96-well plates in DMEM supplemented with $10 \%$ FCS. After $12 \mathrm{~h}$, TEC was starved with medium without FCS for $2 \mathrm{~h}$, stimulated with HLSC EVs and then $10 \mu \mathrm{M}$ BrdU was added overnight. The plates were analyzed by BrdU kit (BrdU; Roche Diagnostics, Mannheim, German) and the absorption values were determined at a $405 \mathrm{~nm}$ wavelength.

In vitro scratch wound-healing assay. HaCaT cells were seeded at a density of $\sim 50 \times 10^{3}$ cells/well in 24-well plates in DMEM supplemented with $10 \%$ FCS. When the cells reached complete confluence, they were starved with medium without FCS overnight. The following day, scratch wounds were created with a sterile tip. Prior to stimulation $(\mathrm{t}=0)$, micrographs of the well were obtained using a Leica microscope (Leica, Wetzlar, Germany). The cells were then stimulated with EVs $(50,000$ EVs/target cells) isolated from the saliva of three different donors. The 'wound closure' phenomenon was monitored for $36 \mathrm{~h}$ using the Leica microscope and images were analyzed by Image J software (Bethesda, MD, USA) observing the decrease of the wound area in cells stimulated with saliva EVs in comparison to cells not stimulated with EVs.

Statistical analysis. Data were presented as mean \pm SD. Statistical analysis was performed using ANOVA with Dunnet's multicomparison tests when appropriate. $\mathrm{P}<0.05$ was considered significant.

\section{Results}

The analysis of the $\zeta$ potential was performed on different biological samples showing that EVs have a negative charge (Table I). In preliminary experiments, serum was incubated with different doses of protamine $(1.0,0.5,0.25$ and $0.1 \mathrm{mg} / \mathrm{ml})$ overnight at $4{ }^{\circ} \mathrm{C}$ and precipitated EVs were recovered by centrifugation at 3,000 $\mathrm{xg}$ for $30 \mathrm{~min}$ (Fig. 1A). However, the EV pellet was easily re-suspended with the dose of $0.25 \mathrm{mg}$ protamine $/ \mathrm{ml}$ serum whereas higher concentrations generated 
A

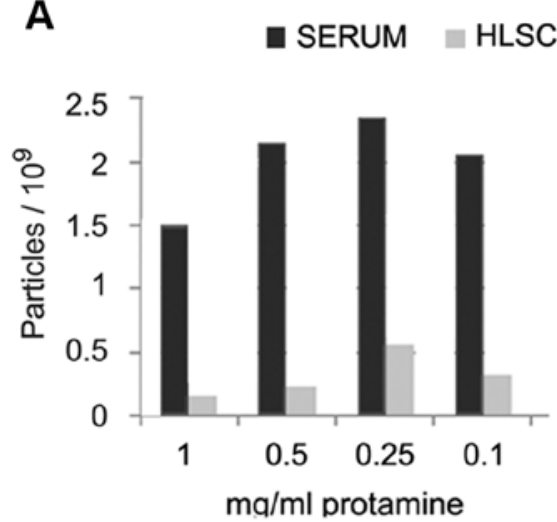

C

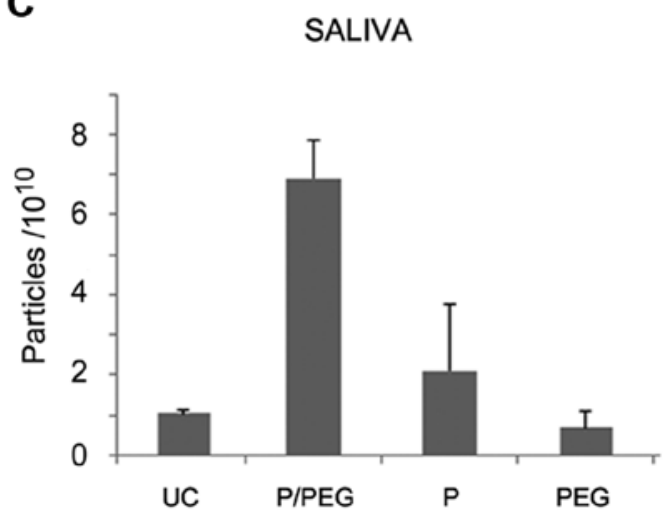

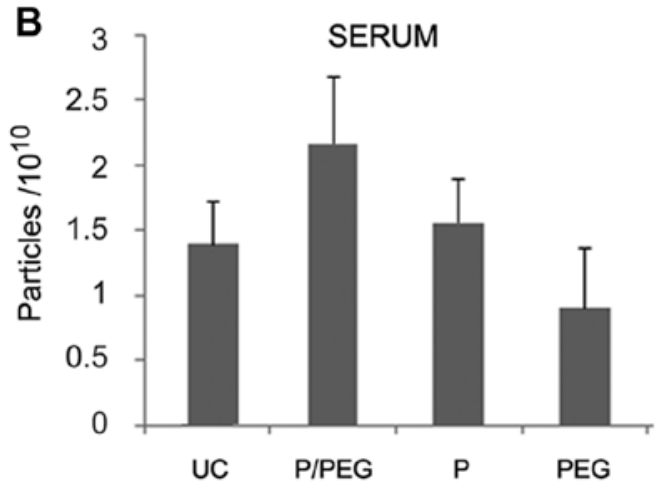

D

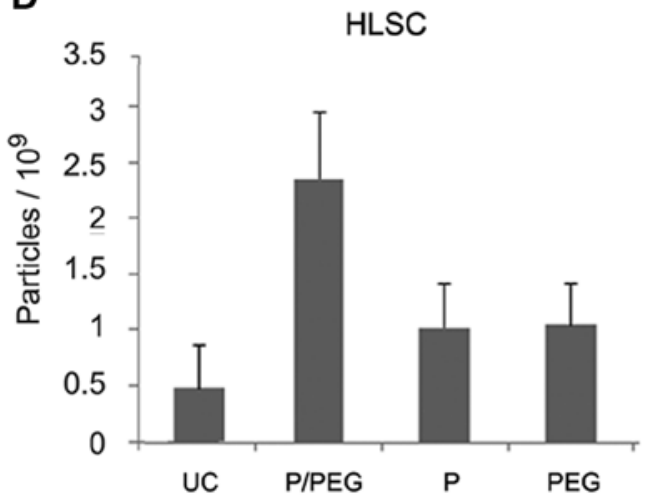

Figure 1. Nanoparticle tracking analysis (NTA) of extracellular vesicles (EVs) isolated from serum, saliva and culture medium of human liver stem cells (HLSCs). (A) Number of particles precipitated from $250 \mu \mathrm{l}$ of serum and $1.5 \mathrm{ml}$ of HLSC culture medium by the addition of different doses of protamine $(1,0.5,0.25$ and $0.1 \mathrm{mg} / \mathrm{ml})$. (B-D) Number of particles isolated from $750 \mu 1$ of serum (B), $2.5 \mathrm{ml}$ saliva (C) and $1.5 \mathrm{ml}$ culture medium of HLSCs (2x10 6 cells; (D) by ultracentrifugation (UC) or P/PEG, $\mathrm{P}$ alone and PEG alone. Data are mean $\pm 1 \mathrm{SD}$ of three independent experiments evaluated in triplicate. ANOVA with Dunnet's multicomparison test was performed all samples vs. UC; ${ }^{\mathrm{P}}<0.05$.

pellets that were more difficult to re-suspend. We observed that the addition of PEG 35,000 Da to protamine favored resuspension. On this basis, a precipitation strategy was established to favor precipitation of negatively charged EVs into a polymeric matrix that would allow the recovery of EVs following centrifugation without the need of an ultracentrifugation step.

Fig. 1 shows the comparison by NTA of EV recovery from serum, saliva and cell free supernatant of HLSCs after ultracentrifugation (UC) or precipitation with P/PEG, PEG alone and protamine alone. The results indicated that $\mathrm{P} / \mathrm{PEG}$ precipitation was more efficient than other conditions in terms of number of EVs detected by NTA. The comparison between serum and saliva of P/PEG-precipitated EVs indicated an enrichment of vesicles in saliva in respect to serum. The size of EVs isolated in the different conditions was similar as observed by transmission electron microscopy. Serum-derived EVs ranged from 35 to $95 \mathrm{~nm}$, whereas those derived from saliva were a more homogeneous population with a size ranging from 45 to $65 \mathrm{~nm}$. EVs derived from HLSCs ranged from 45 to $75 \mathrm{~nm}$ (Fig. 2A).

As for EVs obtained by ultracentrifugation, the western blot analysis of EVs precipitated from serum, saliva and HLSCs by P/PEG showed the expression of CD63,CD9 and CD81 exosomal markers (Fig. 2B). Since it has been suggested that precipitation techniques co-isolate contaminant lipoproteins $(12,13)$, using western blot analysis, we evaluated the presence of ApoB100 and ApoA1 in EVs obtained by ultracentrifugation and P/PEG precipitation. As shown in Fig. 2B, ApoB100 and ApoA1 were detected in serum EVs obtained by ultracentrifugation and precipitation. In saliva EVs, ApoB100 was absent. ApoA1 was detectable in EVs obtained from saliva following ultracentrifugation, whereas ApoA1 was barely detectable in P/PEG precipitation samples. ApoB100 was absent in EVs purified with HLSC culture media by ultracentrifugation and precipitation, whereas ApoA1 was detectable only in EVs purified with ultracentrifugation. To remove lipoproteins, Sephadex G-100 spin columns were used and the EVs were recovered in the void volumes whereas apo-lipoproteins were retained. As shown in Fig. 3A, gel-filtration with Sephadex G-100 reduced ApoB100 and ApoA1 in serum EVs and ApoA1 in saliva EVs.

Detection of RNAs in EVs. As shown in Fig. 3B, the amount of RNA extracted after Sephadex G-100 pre-absorption was reduced in serum and saliva but the difference was not statistically significant $(\mathrm{P}>0.05)$. $\mathrm{PCR}$ analysis showed also a reduction of $\sim 2$ cycles of a representative mRNA present in serum EVs (ID1 mRNA) (Fig. 3C). Fig. 4 shows a comparison between RNA extracted from EVs prepared by $\mathrm{P} / \mathrm{PEG}$ precipitation and ultracentrifugation. No significant difference of RNA content was observed between EVs isolated using the two methods. To evaluate whether RNA extracted from EVs prepared by P/PEG precipitation was suitable for the detection of miRNAs or 
A

UC

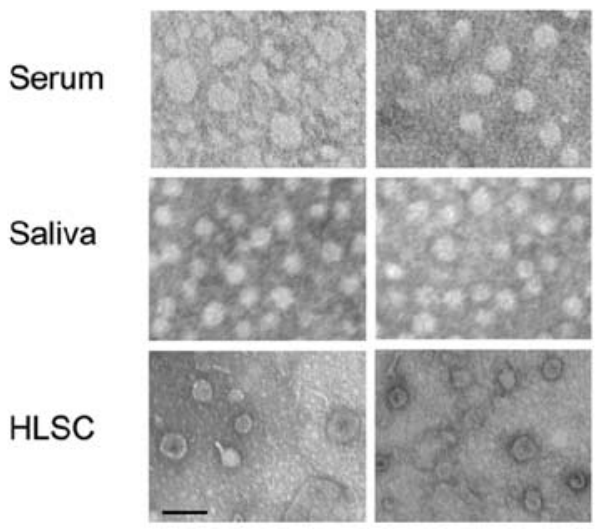

B
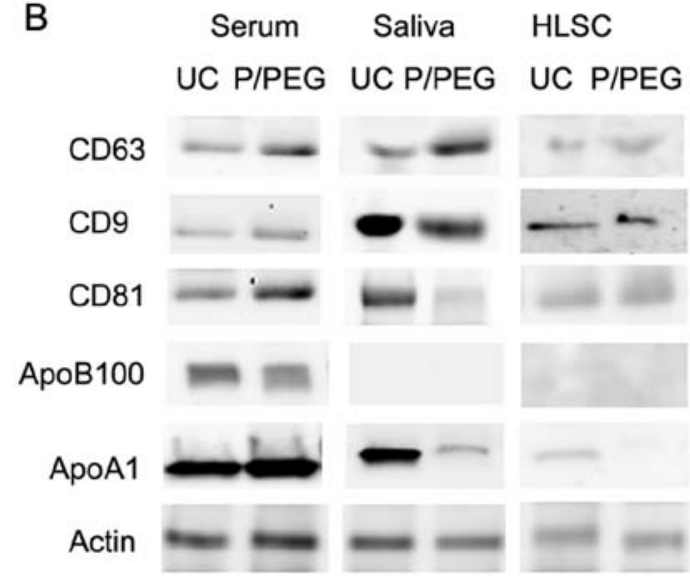

$40 \mathrm{kDa}$

$25 \mathrm{kDa}$

$26 \mathrm{kDa}$

$549 \mathrm{kDa}$

$28 \mathrm{kDa}$

$43 \mathrm{kDa}$

Figure 2. Comparison of extracellular vesicles (EVs) purified by ultracentrifugation and P/PEG precipitation. (A) Representative transmission electron microscopy of EVs isolated by ultracentrifugation (UC) or by P/PEG precipitation and negatively stained with NanoVan. EVs were viewed using a JEOL Jem 1010 electron microscope (black line, $100 \mathrm{~nm}$ ). Three experiments were performed with similar results. (B) Representative western blot analysis of CD63, CD9, CD81 and Actin expression by EVs isolated with UC or by P/PEG precipitation from serum, saliva and human liver stem cell (HLSC) (four experiments were performed with similar results) and of apolipoprotein B100 (ApoB100) and apolipoprotein A1 (ApoA1) associated with EVs (five experiments were performed with similar results).

A
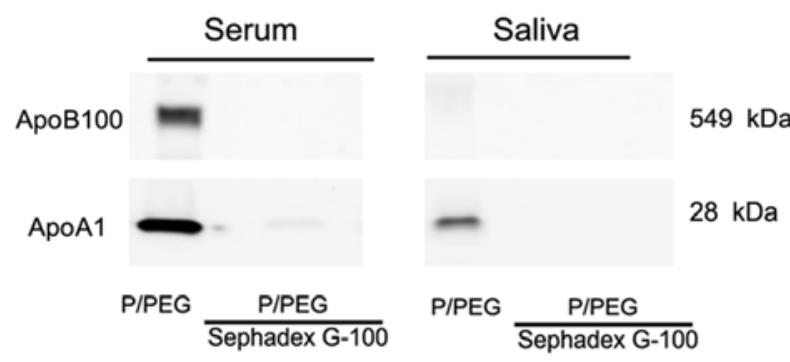

C

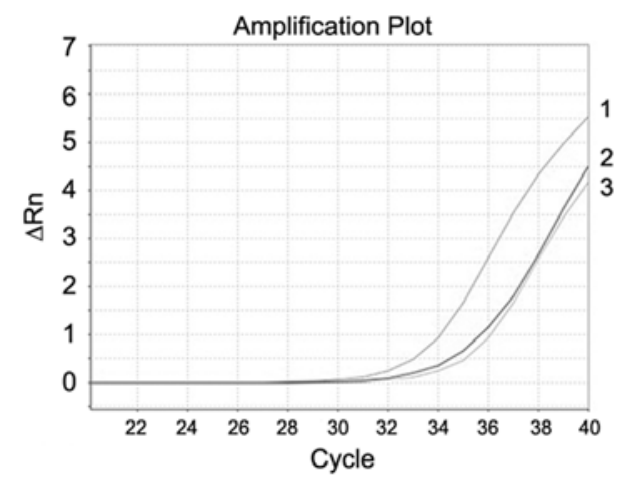

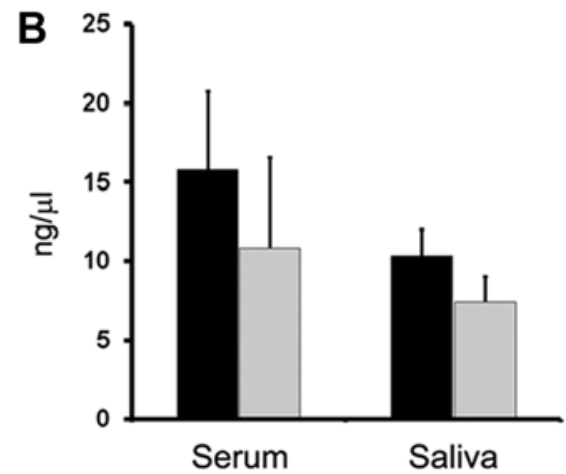

Figure 3. Apolipoprotein is associated with extracellular vesicles (EVs) purified by ultracentrifugation and P/PEG precipitation from serum, saliva and human liver stem cell (HLSC). (A) Representative western blot analysis of apolipoprotein B100 (ApoB100) and apolipoprotein A1 (ApoA1) associated with EVs isolated by P/PEG precipitation from serum and saliva after gel-filtration with Sephadex G-100 spin columns to remove lipoproteins. Two experiments were performed with similar results. (B) Total RNA extraction from EVs separated from serum and saliva before and after gel-filtration with Sephadex G-100 spin columns to remove lipoproteins. Data are mean \pm 1 SD of three independent experiments. (C) Representative PCR analysis for ID1 mRNA expressed by serum EVs before 1) and after gel-filtration 2,3) with Sephadex G-100 spin columns to remove lipoproteins. Two experiments were performed with similar results.

mRNA, RT-qPCR analysis was performed. RT-qPCR analysis revealed the presence of comparable amounts of miR-16, -29a, $-99 \mathrm{~b},-191$ and -223 , in EVs isolated from normal subjects using the two techniques. By contrast, miR-500,-142-3p,-127-3p and -155 were undetectable or detectable at very low levels (not shown). Fig. 4B shows a representative amplification plot for miR-191 in EVs obtained by ultracentrifugation and precipitation. We also performed RT-qPCR analysis for the detection of mRNA. As shown in Fig. 4C, comparable amounts of selected mRNA were detected in EVs derived from serum, saliva and 
A

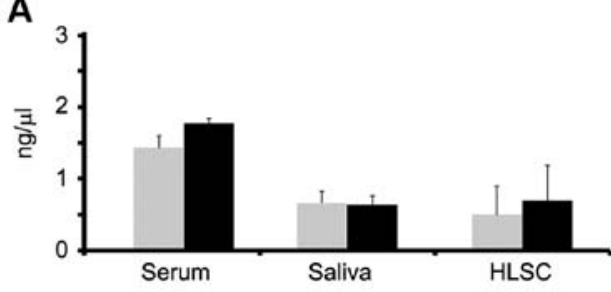

C

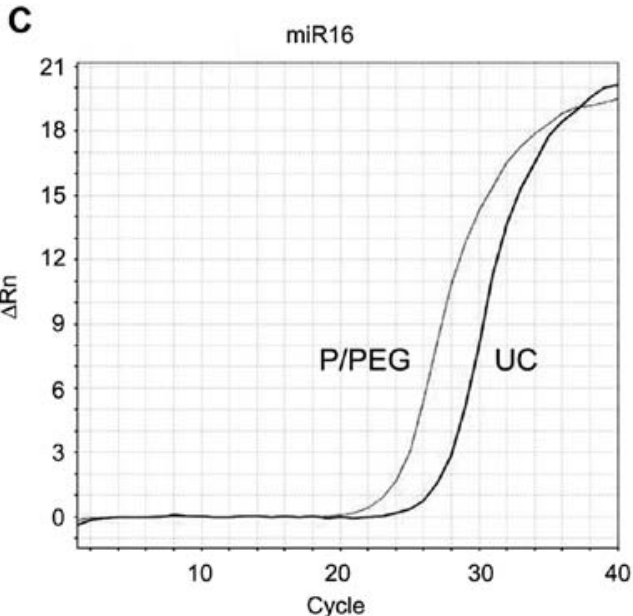

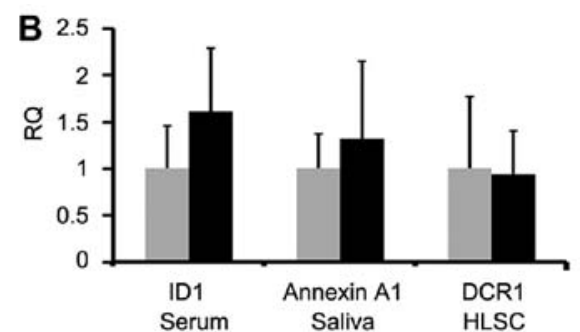

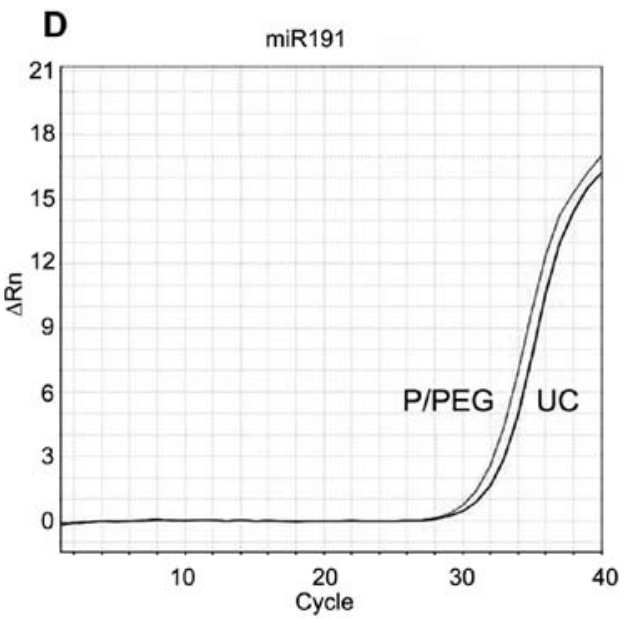

Figure 4. RNA quantification of extracellular vesicles (EVs) purified by ultracentrifugation and P/PEG precipitation from serum, saliva and human liver stem cells (HLSCs) and PCR analysis of mRNA and miRNA. (A) Quantification of total RNA extracted from EVs separated by ultracentrifugation (UC) (gray columns) and P/PEG precipitation (dark columns) from serum, saliva and HLSCs. Data are mean \pm 1 SD of three experiments. (B) RT-qPCR analysis of representative mRNA expressed by serum (ID1), saliva (Annexin A1) and HLSC (DCR1) EVs isolated with UC (grey columns) or P/PEG precipitation (black columns). 18S was used to normalize RNA input and data are expressed as the relative quantification level (RQ) (mean \pm 1 SD of three experiments). (C and D) Representative detection by PCR of (C) miR-16 and (D) miR-191 in EVs isolated from serum by UC or P/PEG precipitation. Similar results were obtained with other miRNAs expressed by serum EVs (miR-29a, -99b and -223; data not shown).

HLSCs, whether purified by ultracentrifugation or P/PEG precipitation.

Evaluation of the ability of EVs isolated by charge-based precipitation to retain biological activities. The biological activity of EVs obtained by ultracentrifugation and by P/PEG precipitation was evaluated for saliva and HLSC EVs.

To examine the biological activity of saliva EVs we performed an in vitro wound-closure assay using human $\mathrm{HaCaT}$ keratinocytes. Saliva EVs obtained by P/PEG induced a significant wound closure comparable to that of EGF (Fig. 5A-E). In particular, precipitated EVs were more effective than EVs obtained by ultracentrifugation (Fig. 5E).

To examine the biological activity of HLSC EVs we performed in vitro proliferation of TEC. Both precipitated and ultra-centrifuged EVs were able to significantly increase cell proliferation (Fig. 5F).

\section{Discussion}

EVs have recently emerged as an important vehicle of information exchange among cells in the body involved in many physiological and pathological processes. Since they retain several molecular markers of the originator cell, EVs isolated from biological fluids may be exploited as a diagnostic tool $(3,6)$. Nevertheless, techniques of EV purification and consequent analysis of EVs remain a challenge. In the present study, we suggest a charge-based precipitation of EVs from biological fluids and cell supernatants. The analysis of $\zeta$ potential revealed that EVs have a negative charge that allows the interaction with a positively charged molecule such as protamine. Protamine was shown to induce EV precipitation from the biological fluids and cell culture media avoiding the ultracentrifugation. When protamine-induced precipitation was performed in a polymeric matrix such as PEG 35,000 Da, the EV recovery was enhanced and pellets were easily re-suspended.

The 'gold standard' methods of EV purification are the differential ultracentrifugation or density gradient ultracentrifugation. These methods, however, are influenced by several parameters that are difficult to standardize, such as viscosity of solutions, rotor type, centrifugal radius and $\mathrm{g}$ force. In addition, the integrity of EVs after prolonged high-speed ultracentrifugation may be damaged. Specifically, membrane debris were observed by electron microscopy and difficulty in recovering RNA and exosomal proteins has been reported (13-16). Several other approaches to EV purification have been investigated. Size exclusion chromatography may have an advantage on ultracentrifugation in maintaining EV integrity since they are not subjected to shear stress (17-19). Filtration with membranes with appropriate pores is also an alternative, but does not guarantee removal of several small contaminants and loss of EVs by binding to membranes (20). The immunoaffinity purification may isolate specific exosome subtypes maintaining integrity of their cargo (16,20-22). A limitation of most of these techniques is the efficiency in the recovery of sufficient amounts of EVs starting from small biological samples. The polymeric precipi- 

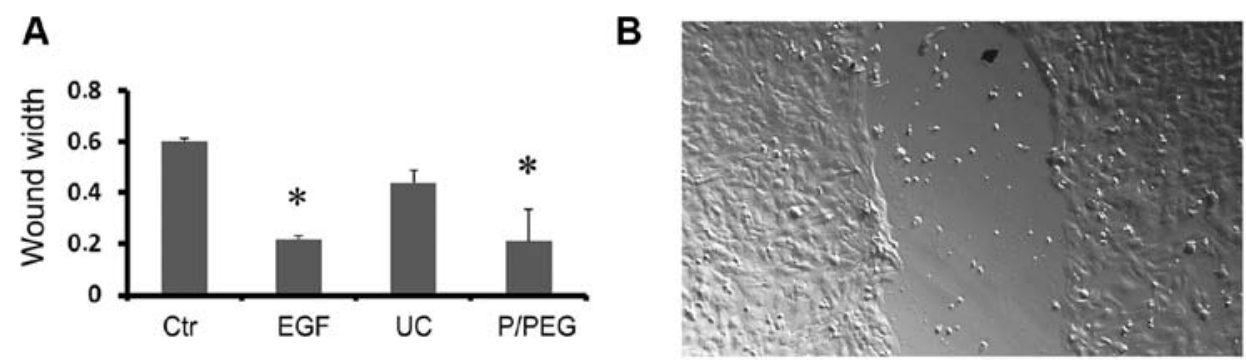

C

D
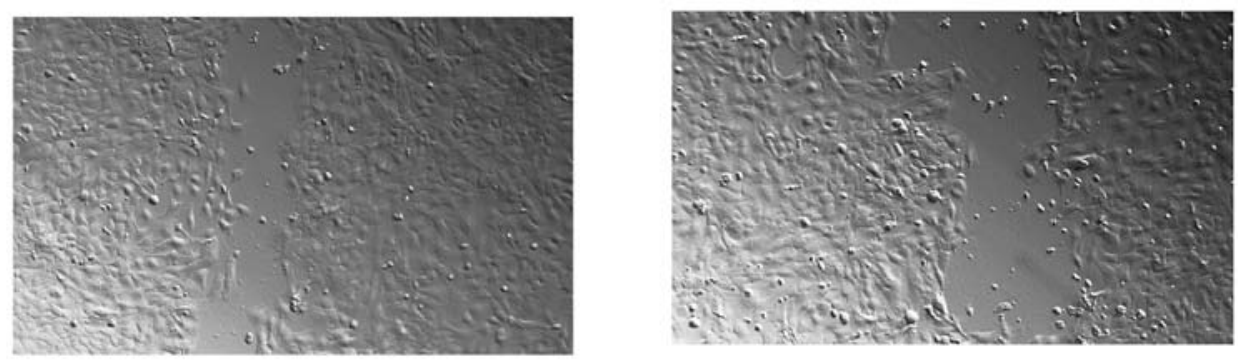

E

F
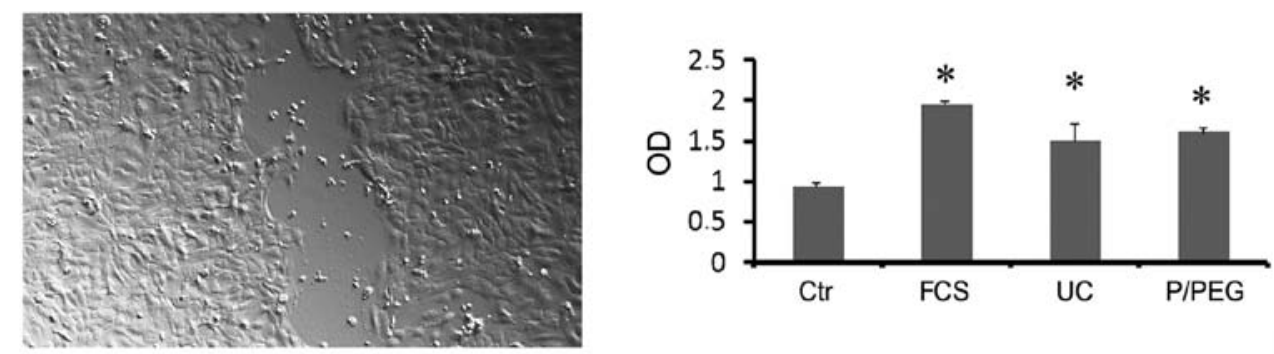

Figure 5. Biological activity of extracellular vesicles (EVs) purified by ultracentrifugation and P/PEG precipitation from saliva and human liver stem cells (HLSCs). (A-E) Evaluation of wound healing on normal dermal keratinocytes (HaCaT) by scratch test. Quantitative evaluation of wound size reduction after 36-h incubation with the vehicle alone (Ctr), $10 \mathrm{ng} / \mathrm{ml}$ EGF as a positive control, and EVs isolated by ultracentrifugation (UC) or P/PEG precipitation (50,000 EVs/cell). Data are mean $\pm 1 \mathrm{SD}$ of three independent experiments evaluated in triplicate. ANOVA with Dunnet's multicomparison test was performed in all the samples vs. UC; ${ }^{*} \mathrm{P}<0.05$. Representative micrographs of Ctr (B), EGF (C), UC EVs (D) and P/PEG EVs (E) induced wound healing. Original magnification, $\mathrm{x} 100$. (F) Proliferation of TEC evaluated by BrdU incorporation after 12-h incubation with EVs isolated by UC or P/PEG precipitation (10,000 EVs/cell). As negative control, TEC was incubated with the vehicle alone in the absence of fetal calf serum (FCS); as positive control, cells were incubated with $10 \%$ FCS. Data are mean \pm 1 SD of three independent experiments evaluated in triplicate. ANOVA with Dunnet's multicomparison test was performed in all the samples versus $\mathrm{Ctr}$; $\mathrm{P}<0.05$.

tation technique, based on the ability of PEG to entrap EVs, has been shown to be a rapid approach to EV isolation from biological samples (5,23-28). This technique has been developed on the observation that PEG allows virus precipitation (23) and several products based on the use of PEG with 8,000 Da molecular weight are commercially available. A recent study suggested heparin affinity chromatography purification of EVs based on the presence of a putative receptor for heparin (29). This technique was shown to allow the purification of EVs with low protein contamination and detection of mRNA from plasma samples in amounts comparable to ultracentrifugation.

In the present study, we combined the charge-based and polymeric precipitation using protamine and PEG 35,000 Da and we compared this technique with differential ultracentrifugation. $\mathrm{P} / \mathrm{PEG}$ was more efficient for the recovery of EVs from small volumes of serum and saliva as well as from the conditioned medium of cultured cells than ultracentrifugation as judged by NTA. The size of vesicles seen by electron microscopy was similar but the membrane debris present in the ultra-centrifuged EVs were absent in the P/PEG EV preparations. In particular, EVs precipitated from saliva were very homogeneous in size and shape. The expression of exosomal markers in EVs obtained by $\mathrm{P} / \mathrm{PEG}$ precipitation as well as the nano-size of vesicles detected by electron microscopy suggest that this method is more suitable for the isolation of small exosomes than of larger shed microvesicles. Since one of the main concerns for EVs obtained by precipitation methods is the presence of contaminants of non-vesicular origin such as lipoproteins $(12,13,30)$, we evaluated the presence of ApoB100 and ApoA1 in the different preparations. The results obtained indicate that in serum EVs, ApoB100 and ApoA1 were present in EV precipitates as well as in EVs purified by differential ultracentrifugation. This may be a limitation for the use of serum EVs for diagnostic purposes if the intent is to discriminate exRNA associated with vesicles from those associated with lipoproteins. However, the detection of exRNA in the biological sample may be exploited for liquid biopsy independently from their vehicle. In this case the precipitation techniques may be suitable for this purpose. ApoB100 was absent in saliva EVs. ApoA1 was present in EV saliva but more expressed in EVs obtained by ultracentrifugation than in P/PEG precipitated EVs. ApoB100 and ApoA1 were barely or absent in EVs purified from culture media by $\mathrm{P} / \mathrm{PEG}$ prepara- 
tions, suggesting that lipoprotein contamination is less relevant for these biological samples.

The most diffuse precipitation method used, ExoQuick, developed by System Biosciences (Mountain View, CA, USA) has solved this problem by a pre-clinical approach to remove lipoproteins. As an alternative, the use of Sephadex G-25 spin columns to remove PEG 8,000 Da containing lipoproteins from precipitated EVs has been suggested (5). Since we precipitated EVs with protamine in association with PEG 35,000 Da, Sephadex G-100 spin columns were used to show the effective reduction of apo-lipoprotein contaminants. Following absorption, the total RNA was reduced but was suitable for the detection of miRNA and mRNA content of EVs.

EVs obtained by P/PEG precipitation retained in vitro the biological activity as seen by the induction of wound closure by keratinocytes stimulated with EVs from saliva and of proliferation of TEC challenged with EVs released by HLSCs.

The methods currently available for EV purification have both advantages and disadvantages and possibly none are ideal for each application $(5,6,31)$. The methods described in the present study have the merit of simplicity and avoid requirement of expensive equipments. In addition, the isolated EVs retained the biological activities.

In conclusion, we have shown that charge-based precipitation of EVs may be used for an efficient isolation of EVs from biological samples and may be exploited for the search of new biomarkers.

\section{Acknowledgements}

The present study was supported by a grant from Unicyte, SW. M.C.D., F.F., M.F.B., C.T. and G.C. are named as inventors in EV-related patents. C.T. is a full-time employee of Fresenius Medical Care and contributed to the study as researcher.

\section{References}

1. Ratajczak J, Wysoczynski M, Hayek F, Janowska-Wieczorek A and Ratajczak MZ: Membrane-derived microvesicles: Important and underappreciated mediators of cell-to-cell communication. Leukemia 20: 1487-1495, 2006.

2. Cocucci E, Racchetti G and Meldolesi J: Shedding microvesicles: Artefacts no more. Trends Cell Biol 19: 43-51, 2009.

3. Quesenberry PJ, Aliotta J, Deregibus MC and Camussi G: Role of extracellular RNA-carrying vesicles in cell differentiation and reprogramming. Stem Cell Res Ther 6: 153, 2015.

4. Shelke GV, Lässer C, Gho YS and Lötvall J: Importance of exosome depletion protocols to eliminate functional and RNA-containing extracellular vesicles from fetal bovine serum. J Extracell Vesicles: Sep 30, 2014 (Epub ahead of print). doi: $10.3402 /$ jev.v3.24783

5. Szatanek R, Baran J, Siedlar M and Baj-Krzyworzeka M: Isolation of extracellular vesicles: Determining the correct approach (Review). Int J Mol Med 36: 11-17, 2015.

6. Taylor DD, Zacharias W and Gercel-Taylor C: Exosome isolation for proteomic analyses and RNA profiling. Methods Mol Biol 728: 235-246, 2011.

7. Herrera MB, Bruno S, Buttiglieri S, Tetta C, Gatti S, Deregibus MC Bussolati B and Camussi G: Isolation and characterization of a stem cell population from adult human liver. Stem Cells 24: 2840-2850, 2006.

8. Herrera MB, Fonsato V, Gatti S, Deregibus MC, Sordi A, Cantarella D, Calogero R, Bussolati B, Tetta C and Camussi G: Human liver stem cell-derived microvesicles accelerate hepatic regeneration in hepatectomized rats. J Cell Mol Med 14 $1605-1618,2010$.

9. Cantaluppi V, Biancone L, Romanazzi GM, Figliolini F, Beltramo S, Galimi F, Camboni MG, Deriu E, Conaldi P, Bottelli A, et al: Macrophage stimulating protein may promote tubular regeneration after acute injury. J Am Soc Nephrol 19: 1904-1918, 2008.
10. Théry C, Amigorena S, Raposo G and Clayton A: Isolation and characterization of exosomes from cell culture supernatants and biological fluids. Curr Protoc Cell Biol: Apr 1, 2006. (Epub ahead of print). doi: 10.1002/0471143030.cb0322s30.

11. Iavello A, Frech VS, Gai C, Deregibus MC, Quesenberry PJ and Camussi G: Role of Alix in miRNA packaging during extracellular vesicle biogenesis. Int J Mol Med 37: 958-966, 2016.

12. Witwer KW, Buzás EI, Bemis LT, Bora A, Lässer C, Lötvall J, Nolte't Hoen EN, Piper MG, Sivaraman S, Skog J, et al: Standardization of sample collection, isolation and analysis methods in extracellular vesicle research. J Extracell Vesicles 2: 20360, 2013.

13. Momen-Heravi F, Balaj L, Alian S, Trachtenberg AJ, Hochberg FH, Skog J and Kuo WP: Impact of biofluid viscosity on size and sedimentation efficiency of the isolated microvesicles. Front Physiol 3: 162, 2012.

14. Jeppesen DK, Hvam ML, Primdahl-Bengtson B, Boysen AT, Whitehead B, Dyrskjøt L, Orntoft TF, Howard KA and Ostenfeld MS: Comparative analysis of discrete exosome fractions obtained by differential centrifugation. J Extracell Vesicles 3: 25011, 2014.

15. Cvjetkovic A, Lötvall J and Lässer C: The influence of rotor type and centrifugation time on the yield and purity of extracellular vesicles. J Extracell Vesicles 3: 3, 2014.

16. Tauro BJ, Greening DW, Mathias RA, Ji H, Mathivanan S, Scott AM and Simpson RJ: Comparison of ultracentrifugation, density gradient separation, and immunoaffinity capture methods for isolating human colon cancer cell line LIM1863-derived exosomes. Methods 56: 293-304, 2012.

17. Müller G: Novel tools study cell type-specific exosomes microvesicles. J Bioanal Biomed 4: 46-60, 2012.

18. Taylor DD, Lyons KS and Gerçel-Taylor C: Shed membrane fragment-associated markers for endometrial and ovarian cancers. Gynecol Oncol 84: 443-448, 2002.

19. Böing AN, van der Pol E, Grootemaat AE, Coumans FA, Sturk A and Nieuwland R: Single-step isolation of extracellular vesicles by size-exclusion chromatography. J Extracell Vesicles 3: 3, 2014.

20. Taylor DD and Shah S: Methods of isolating extracellular vesicles impact down-stream analyses of their cargoes. Methods 87: 3-10, 2015.

21. Caby MP, Lankar D, Vincendeau-Scherrer C, Raposo G and Bonnerot C: Exosomal-like vesicles are present in human blood plasma. Int Immunol 17: 879-887, 2005

22. Zarovni N, Corrado A, Guazzi P, Zocco D, Lari E, Radano G, Muhhina J, Fondelli C, Gavrilova J and Chiesi A: Integrated isolation and quantitative analysis of exosome shuttled proteins and nucleic acids using immunocapture approaches. Methods 87 : 46-58, 2015.

23. Hebert TT: Precipitation of plant viruses by polyethylene glycol. Phytopathology 53: 362, 1963.

24. Alvarez ML, Khosroheidari M, Kanchi Ravi R and DiStefano JK: Comparison of protein, microRNA, and mRNA yields using different methods of urinary exosome isolation for the discovery of kidney disease biomarkers. Kidney Int 82: 1024-1032, 2012.

25. Alvarez ML: Isolation of urinary exosomes for RNA biomarker discovery using a simple, fast, and highly scalable method. Methods Mol Biol 1182: 145-170, 2014.

26. Rekker K, Saare M, Roost AM, Kubo AL, Zarovni N, Chiesi A, Salumets A and Peters M: Comparison of serum exosome isolation methods for microRNA profiling. Clin Biochem 47: $135-138,2014$.

27. Zlotogorski-Hurvitz A, Dayan D, Chaushu G, Korvala J, Salo T, Sormunen R and Vered M: Human saliva-derived exosomes: Comparing methods of isolation. J Histochem Cytochem 63: 181-189, 2015.

28. Kanchi Ravi R, Khosroheidari M and DiStefano JK: A modified precipitation method to isolate urinary exosomes. J Vis Exp 95: 51158, 2015.

29. Balaj L, Atai NA, Chen W, Mu D, Tannous BA, Breakefield XO, Skog J and Maguire CA: Heparin affinity purification of extracellular vesicles. Sci Rep 5: 10266, 2015.

30. Yuana Y, Levels J, Grootemaat A, Sturk A and Nieuwland R: Co-isolation of extracellular vesicles and high-density lipoproteins using density gradient ultracentrifugation. J Extracell Vesicles 3: 3, 2014

31. Taylor DD: Isolation and molecular characterization of extracellular vesicles. Methods 87: 1-2, 2015. 